\title{
Two Methods of Proving the Improved Mean Value Theorem of Integral
}

\author{
Pei Hong Mei* \\ Department of Basis \\ Dalian Naval Academy \\ Dalian, China \\ E-mail: hongmei_005@163.com \\ * Corresponding Author \\ Shang Jie Lin \\ Department of Basis \\ Dalian Naval Academy \\ Dalian, China \\ E-mail: 343065787@qq.com
}

\author{
Li Xuan Hai \\ Department of Basis \\ Dalian Naval Academy \\ Dalian, China \\ E-mail: 1638914812@qq.com
}

\begin{abstract}
The proof of the mean value theorem for integral, which is given by Advanced Mathematics and which is wildly used, only proved that the mean value $\xi$ is on the closed interval $[a, b]$. In this paper, we provide two different methods for the proof of the mean value theorem of integral and prove the mean value $\xi$ is in the open interval $(a, b)$, which is an improvement in the conclusion of the theorem. In the end, we illuminate the practicability of the improved mean value theorem for integral with two examples as follows.
\end{abstract}

Keywords- Mean Value Theorem of Integral; Mean Value Theorem of Differential; Improved; Maximum and Minimum Value; Comparison Property

\section{INTRODUCTION}

The mean value theorem of integral is an important theorem of calculus, together with the mean value theorem of differential, which constitutes the theoretical basis of Advanced Mathematics. The relationships between them had been widely researched and discussed ${ }^{[1-3]}$. In the Advanced Mathematics ${ }^{[4]}$, the traditional method to prove the mean value theorem of integral is using the intermediate value theorem of continuous functions, having a flaw in it which has caused a widely discuss and research ${ }^{[5-10]}$. On the basis of discussion, we improved the conclusion of mean value theorem by using two methods in this article.

\section{PRELIMINARY}

First we give some basic theorems we will need as follows. The proof of the theorems sees [4].

Boundedness and Max-min Theorem:

If $f(x)$ is a continuous function on a closed interval, then $f(x)$ has an absolute maximum and an absolute minimum on the interval. In particular, $f(x)$ must be bounded on the interval.

\section{The Zero Point Theorem:}

Let $f(x)$ be a continuous function on the interval $[a, b]$ and $f(a) \cdot f(b)<0$, then there is a $\xi \in(a, b)$, such that

$$
f(\xi)=0
$$

\section{Fundamental Theorem of Calculus}

Let $f(x)$ be continuous (hence integrable) on $[a, b]$, and let $F(x)$ be any antiderivative of $f(x)$ on $[a, b]$. Then

$$
\int_{a}^{b} f(x) d x=F(b)-F(a)
$$

\section{The Intermediate Value Theorem}

Let $f(x)$ be a continuous function on the interval $[a, b]$ and let $C$ be a number between $f(a)=A$ and $f(b)=B$, then there is at least one number $\xi \in(a, b)$, such that

$$
f(\xi)=C,(a<\xi<b) .
$$

Mean Value Theorem of Differentials:

If $f(x)$ is continuous on the closed interval $[a, b]$ and differentiable in the open interval $(a, b)$, then there is at least one number $\xi$ in $(a, b)$ where

$$
\frac{f(b)-f(a)}{b-a}=f^{\prime}(\xi) \quad(a \leq \xi \leq b),
$$

Or equivalently, where

$$
f(b)-f(a)=f^{\prime}(\xi)(b-a) \quad(a \leq \xi \leq b) .
$$


In the mean value theorem for differential, $\xi \in(a, b)$, so if we can prove the mean theorem for integral by using it, then the point that in the conclusion can be limited within the open interval $(a, b)$. We will give the prove process below.

\section{Mean Value Theorem of Integral:}

If $f(x)$ is continuous on $[a, b]$, there is at least one number $\xi$ on the interval $[a, b]$ such that

$$
\int_{a}^{b} f(x) \mathrm{d} x=f(\xi)(b-a) \quad(a \leq \xi \leq b) .
$$

For the prove process, please refer the documentation for information ${ }^{[4]}$. In the conclusion of the theorem, $\xi$ 's value range is closed interval $[a, b]$, however, if its value range were open interval $(a, b)$, then the theorem will be much more widely used. In fact it can be proved that the mean value $\xi$ is within open interval $(a, b)$, that is to say the mean value theorem for interval can be improved to be the conclusion as follows

\section{MAIN RESULTS}

\section{A. Improved Mean Value Theorem of Integrals}

Improved Mean Value Theorem of Integral:

If $f(x)$ is continuous on the closed interval $[a, b]$, then there is at least one number $\xi$ in the interval $(a, b)$ such that

$$
\int_{a}^{b} f(x) \mathrm{d} x=f(\xi)(b-a) \quad(a<\xi<b) .
$$

\section{B. Use the Mean Value Theorem of Differentials to Prove the Mean Value Theorem of Integral}

From the fundamental theorem of calculus, we can find the relationships between definite integral and the primitive function of integrand, thus we can use the mean value theorem for differentials to prove the mean value theorem for integral. We have provided the mean value theorem for differentials, which is also named Lagrange mean value theorem above. Now we give the prove process as below:

Proof Since $f(x)$ is continuous on the closed interval $[a, b]$, it has the primitive functions.

Let $F(x)$ be any primitive function of $f(x)$ on $[a, b]$, so $F^{\prime}(x)=f(x)$ on the closed interval $[a, b]$. According to Newton-Leibniz formula, we can get

$$
\int_{a}^{b} f(x) \mathrm{d} x=F(b)-F(a)
$$

As function $F(x)$ is derivable in $[a, b]$, according to the relationships between the derivable and continuous, we can know that $F(x)$ is continuous in $[a, b]$, thus $F(x)$ satisfies the conditions of the mean value theorem for differential, and according to the mean value theorem for differential, there is at least one point $\xi$ exists within open interval $(a, b)$ to make that

$$
\begin{gathered}
F(b)-F(a)=F^{\prime}(\xi)(b-a) \\
=f(\xi)(b-a), \\
\xi \in(a, b),
\end{gathered}
$$

Therefore

$$
\int_{a}^{b} f(x) \mathrm{d} x=f(\xi)(b-a), \quad \xi \in(a, b) .
$$

From that the mean value theorem for integral process has been completed, we limited the value range of the point $\xi$ within an open interval $(a, b)$. However, NewtonLeibniz formula was used in this prove process, while in the Advanced Mathematics textbook the mean value theorem for integral appears earlier than Newton-Leibniz formula, so in the textbook, the method above was not used to prove the mean value theorem of integral, instead the maximum and minimum value of a continuous function and definite integral's comparative property was used to prove it, so the conclusion $\xi \in[a, b]$ was got. In fact, $\xi \in(a, b)$ still can be proved by improving this prove method, we will show you the improved prove method as below.

\section{Use Maximum and Minimum of Function and Comparison Property of Definite Integral to Prove the Mean Value Theorem of Integrals}

Theorem If $f(x), g(x)$ are continuous on the closed interval $[a, b]$. If on the closed interval $[a, b] f(x) \leq g(x)$, and moreover $f(x) \not \equiv g(x)$, then

$$
\int_{a}^{b} f(x) \mathrm{d} x<\int_{a}^{b} g(x) \mathrm{d} x
$$

Proof Assume that

$$
F(x)=g(x)-f(x), x \in[a, b]
$$

we can get that $F(x)$ continuous on interval $[a, b]$, and $F(x) \geq 0$, but $F(x) \not \equiv$, so there must have a $x_{0} \in[a, b]$, to make $F\left(x_{0}\right)>0$.

As $F(x)$ is continuous on $x=x_{0}$, so there must exist

$$
a \leq \alpha<\beta \leq b,
$$

to establishes

$$
F(x) \geq \frac{F\left(x_{0}\right)}{2}
$$

under the condition $x \in[\alpha, \beta]$, therefore 


$$
\int_{a}^{b} F(x) \mathrm{d} x=\int_{a}^{\alpha} F(x) \mathrm{d} x+\int_{a}^{\beta} F(x) \mathrm{d} x+\int_{\beta}^{b} F(x) \mathrm{d} x
$$

According to the nature of definite integral, we can learn that

$$
\begin{aligned}
& \int_{a}^{\alpha} F(x) \mathrm{d} x \geq 0 \\
& \int_{a}^{\beta} F(x) \mathrm{d} x \geq \int_{a}^{\beta} \frac{F\left(x_{0}\right)}{2} \mathrm{~d} x=\frac{(\beta-\alpha) F\left(x_{0}\right)}{2}>0, \\
& \int_{\beta}^{b} F(x) \mathrm{d} x \geq 0
\end{aligned}
$$

\section{Therefore}

$$
\int_{a}^{b} F(x) \mathrm{d} x=\int_{a}^{\alpha} F(x) \mathrm{d} x+\int_{a}^{\beta} F(x) \mathrm{d} x+\int_{\beta}^{b} F(x) \mathrm{d} x>0,
$$

That is to say that

$$
\int_{a}^{b}[g(x)-f(x)] \mathrm{d} x>0
$$

Thus the conclusion $\int_{a}^{b} f(x) \mathrm{d} x<\int_{a}^{b} g(x) \mathrm{d} x$ proved.

Below we will use above theorem to prove the improved the mean value theorem for integral

Proof As $f(x)$ is continuous on the closed interval $[a, b]$, using the boundedness property and maximum and minimum value theorem of continuous functions on a closed interval $[a, b]$, we can learn that $f(x)$ has an absolute maximum and an absolute minimum on the interval $[a, b]$. Assume the maximum is $M$ and the minimum is $m$, then for any $x$ on the closed interval $[a, b]$, we have

$$
m \leq f(x) \leq M
$$

(1) If $f(x) \equiv C$ ( $C$ is a constant), then

$$
\int_{a}^{b} f(x) \mathrm{d} x=C(b-a)=f(x)(b-a),
$$

So we could let any value in $(a, b)$ be $\xi$. The theorem is proved.

(2) If $f(x) \neq C$ ( $C$ is a constant), then $M \neq m$, and moreover

but

$$
M-f(x) \geq 0
$$

$$
M-f(x) \equiv 0,
$$

according to theorem, we can learn that

$$
\int_{a}^{b} f(x) \mathrm{d} x<M(b-a)
$$

Prove as above, we will get

$$
\int_{a}^{b} f(x) \mathrm{d} x>m(b-a)
$$

So

$$
m(b-a)<\int_{a}^{b} f(x) \mathrm{d} x<M(b-a)
$$

That is to say

$$
m<\frac{\int_{a}^{b} f(x) \mathrm{d} x}{b-a}<M .
$$

According to the intermediate value theorem of continuous functions on a closed interval, we can learn that there is a mean value $\xi$ belonging to the open interval $(a, b)$, which establishes

$$
\int_{a}^{b} f(x) \mathrm{d} x=f(\xi)(b-a)
$$

In a word, we proved the improved mean value theorem of integrals.

\section{APPLICATIONS}

The improved mean value theorem of integral is more flexible. Now we illuminate the practicability of the improved mean value theorem for integral with two examples as follows.

A. Prove $\lim _{n \rightarrow \infty} \int_{0}^{\frac{\pi}{2}} \sin ^{n} x d x=0$.

Proof (1) (Traditional method)

For $\forall \varepsilon>0$, we have

$$
\begin{gathered}
0<\int_{0}^{\frac{\pi}{2}-\varepsilon} \sin ^{n} x d x<\frac{\pi}{2}\left[\sin \left(\frac{\pi}{2}-\varepsilon\right)\right]^{n}, \\
0<\int_{\frac{\pi}{2}-\varepsilon}^{\frac{\pi}{2}} \sin ^{n} x d x<1 \cdot\left[\frac{\pi}{2}-\left(\frac{\pi}{2}-\varepsilon\right)\right]=\varepsilon,
\end{gathered}
$$

So

$$
0<\int_{0}^{\frac{\pi}{2}} \sin ^{n} x d x<\frac{\pi}{2}\left[\sin \left(\frac{\pi}{2}-\varepsilon\right)\right]^{n}+\varepsilon<2 \varepsilon
$$

( $n$ is sufficiently large), 
that is, when $n$ is large enough, we will obtain

$$
0<\int_{0}^{\frac{\pi}{2}} \sin ^{n} x d x<2 \varepsilon
$$

On the basis of the definition of the limit of sequence, we obtain

$$
\lim _{n \rightarrow \infty} \int_{0}^{\frac{\pi}{2}} \sin ^{n} x d x=0
$$

(2) (Prove this problem on the basis of the mean value theorem of integral) obtain

Based on the mean value theorem of integral, we

$$
\begin{gathered}
\int_{0}^{\frac{\pi}{2}} \sin ^{n} x d x=\frac{\pi}{2} \sin ^{n} \xi, \\
\left(0<\xi<\frac{\pi}{2}\right),
\end{gathered}
$$

For we have $\sin \xi<1$, and hence

$$
\sin ^{n} \xi \rightarrow 0,(n \rightarrow \infty)
$$

So we have

$$
\lim _{n \rightarrow \infty} \int_{0}^{\frac{\pi}{2}} \sin ^{n} x d x=\lim _{n \rightarrow \infty} \frac{\pi}{2} \sin ^{n} \xi=0 .
$$

It is obviously that the second method, which proves the problem on the basis of the improved mean value theorem of integral, is simpler. Sometimes the improved mean value theorem of integral can solve the problem while the original mean value theorem of integral cannot. We will give such an example as follow.

\section{B. Prove}

If $f(x) \neq 0$ on the interval $[a, b]$, and $f^{\prime}(x)$ is continuous with $f(a)=0$. Prove that there is a $\xi \in(a, b)$, such that

$$
\left|f^{\prime}(\xi)\right|>\frac{1}{(b-a)^{2}} \int_{a}^{b} f(x) d x .
$$

Proof (1) When $\int_{a}^{b} f(x) d x<0$, for every $x \in(a, b)$, we have

$$
\left|f^{\prime}(x)\right|>\frac{1}{(b-a)^{2}} \int_{a}^{b} f(x) d x .
$$

So we could let any value in $(a, b)$ be $\xi$.
(2) When $\int_{a}^{b} f(x) d x=0$, on the basis of the conditions above, there must be a $x_{0} \in(a, b)$, which satisfies such conditions

$$
f^{\prime}\left(x_{0}\right) \not \equiv 0
$$

On the contrary, if $f^{\prime}(x) \equiv 0, x \in(a, b)$, then $f(x) \equiv C,(x \in[a, b])$, and therefore $f(x) \equiv 0, x \in[a, b]$, which is contradictory with the conditions.

Let $\xi=x_{0}$ and the conclusion is proved.

(3) When $\int_{a}^{b} f(x) d x>0$, since

$$
\begin{aligned}
& \frac{1}{(b-a)} \int_{a}^{b} f(x) d x=f(\eta) \quad(\eta \in(a, b)) \\
&=f(\eta)-f(a) \\
&=f^{\prime}(\xi)(\eta-a) \\
&(\xi \in(a, \eta) \subset(a, b))
\end{aligned}
$$

then

$$
\begin{aligned}
f^{\prime}(\xi) & =\frac{1}{(b-a)(\eta-a)} \int_{a}^{b} f(x) d x \\
& >\frac{1}{(b-a)^{2}} \int_{a}^{b} f(x) d x .
\end{aligned}
$$

So we obtain

$$
\left|f^{\prime}(\xi)\right|>\frac{1}{(b-a)^{2}} \int_{a}^{b} f(x) d x .
$$

It is obviously the original mean value theorem of integral can solve this problem.

\section{CONCLUSIONS}

According to the two proof methods above, we improved the conclusion of the mean value theorem of integral, and limited the mean value $\xi$ from the closed interval $[a, b]$ to the open interval $(a, b)$, which has more flexible application. At last, we illuminated the practicability of the improved mean value theorem of integral with two examples above, which showed the method based on the improved mean value theorem of integral simpler.

\section{ACKNOWLEDGMENT}

This work was supported by the Dalian Naval Academy for Basic Research. 


\section{REFERENCES}

[1] Li Hai-xia, The relevance of differential mean-value theorem and integral mean-value theorem[J], Journal of Nanyang Normal University, 2013, 12(12): 66-68.

[2] Pang Jin-biao, Hou Wei-gen, Zheng Jing-bo, Relations between differential and integral mean value theorem[J], Studies in College Mathematics, 2014, 17(6): 44-46.

[3] Ding Dian-kun, Ma Fang-fang, New method to proof primacy fundamental theorem of differential coefficient and mean value theorem of integral[J], Journal of Qiqihar University, 2007, 23(3): 58-60.

[4] Department of mathematics, Tongji University, Advanced Mathematics[M], The Seventh Edition. Beijing: Higher Education Press, 2014.

[5] Wang Fan-bin, Comfirmation of integral mean value theorem by Cauchy mean value theorem[J], Journal of Neijiang Normal University, 2010, 25(12): 11-14.
[6] Lan Yong-yi, Further discussion of integral mean value theorem[J], Journal of Ningde Teachers College (Natural Science), 2009, 21(3): 233-235.

[7] Yan Yuan-chao, Improvement of the First Integral Mean Value Theorem [J], Journal of Lüliang University, 2012, 2(2): 21-23.

[8] Yu Xian-zhi, Xiao Guang-qiang, Zhao Sheng-li, New Prove of Interior Point Property for Two Integral Mean Value Theorems [J], Journal of Logistical Engineering University, 2013, 29(2): 88-91.

[9] Ding Dian-kun, Zou Yu-mei, Differential Coefficient Median Theorem and Newton-Leibniz Formula Can Be Proved Each Other[J], College Mathematics, 2005, 21(4): 128-130.

[10] YIN Feng, WANG Peng-fei, The Extension of Double Integral Mean Value Theorem [J], Journal of Xinzhou Teachers University, 2011, 27(2): 15-16, 30. 